\title{
Method for Detecting Railway Line Sections Exposed to Strong Winds Using Numerical Simulations
}

\author{
Keiji ARAKI \\ Assistant Senior Researcher, \\ Takaaki FUKUHARA \\ Assistant Senior Researcher, \\ Meteorological Disaster Prevention Laboratory, Disaster Prevention Technology Division \\ Taisuke SHIMAMURA \\ Manager, \\ Research and Development Promotion Division \\ Toshiaki IMAI \\ Laboratory Head, \\ Meteorological Disaster Prevention Laboratory, Disaster Prevention Technology Division \\ The safety of railway line sections against strong winds can be improved by setting up \\ anemometers in locations where wind speeds frequently exceed the critical wind speed of \\ overturning. In order to ensure optimum location of the anemometers, wind speed values \\ need to be estimated over an $N$-year return period along railway lines. This paper intro- \\ duces a method to estimate over a given return period the values of the maximum instan- \\ taneous wind-velocity along railway lines at interval of $100 \mathrm{~m}$, by using two kinds of nu- \\ merical simulation techniques (the meteorological model and the Computational Fluid \\ Dynamics model) and a topographical factor analysis.
}

Keywords: strong wind sections, numerical simulations, topographical factor analysis, value for return period

\section{Introduction}

Anemometers are arranged along operational sections of railway line to prevent accidents with trains overturning due to strong winds. Train dispatchers issue operational controls when anemometers detect wind speeds which exceed the wind speed for operation control.

Railway operation safety against strong winds can be improved by positioning anemometers in locations where wind velocities frequently exceed the critical wind speed of overturning. In order to ensure that anemometers are placed in the optimum position, wind-velocity values along the railway lines in question needs to be estimated over a return period of $N$-year.

This paper introduces a method to estimate these values over a given return period near the surface of the ground using numerical simulations and topographical factor analysis.

\section{Outline of the method for detecting strong wind sections along railway lines}

Generally, accidents involving the overturning of a train are understood to be related not to a mean windvelocity averaged out over a long evaluation period, but rather to instantaneous wind-velocity exceeding a critical value. This study therefore aimed to detect these strong wind sections along railway lines, based on maximum instantaneous wind-velocity values over a given

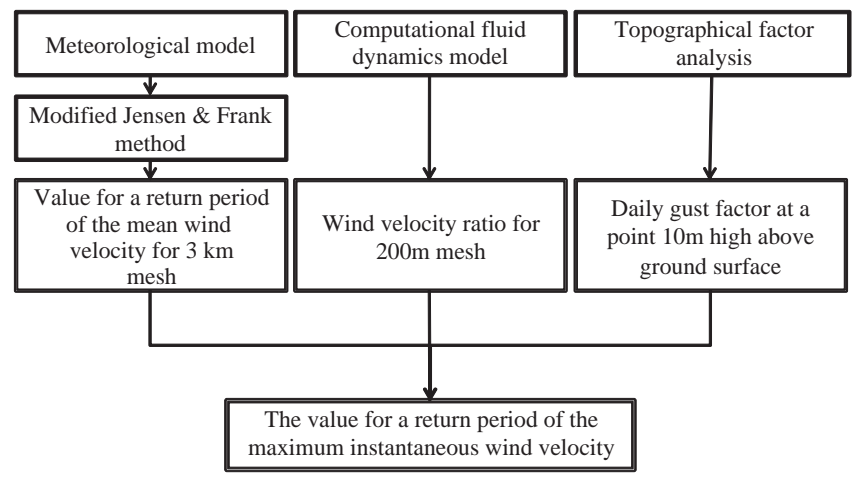

Fig. 1 Flowchart representing the detection of strong wind sections along railway lines

return period and on the fact that the critical wind speeds for overturning have different values depending on the shape of railway structures. However, maximum instantaneous wind-velocity values for a given return period cannot be calculated directly using numerical simulation models. For this reason, separate calculations were made of mean wind-velocity values for a return period and gust factors (ratio of the maximum instantaneous wind velocities to the mean wind velocities), which in turn were then used to obtain maximum instantaneous wind- velocity values over a given return period. Figure 1 is a flowchart illustrating the method used to detect strong wind sections along railway lines, proposed in this study. 
3. Estimation of mean wind-velocity values for a given return period using a meteorological model

\subsection{Meteorological model}

\subsubsection{Overview of the meteorological model}

The meteorological model calculates evolution of metrological elements over time (e.g. atmospheric pressure, atmospheric temperature, wind direction, and wind-velocity) at each grid point of the computational domain, delimited by a three dimensional computational grid. The LOCALS meteorological model by CRC Solutions Ltd. (now ITOCHU Techno-Solutions Corporation Ltd.) [1] was adopted for this study. LOCALS can accurately reproduce geographical features in Japan. Moreover, it includes a feature for reproducing the precipitation process matching the weather in and around Japan. The basic equation system is based on the hydrostatic and completely compressive equation system, which assumes that atmospheric movement in the vertical direction is much smaller than in the horizontal direction. The computational domain terrain was reproduced with $3 \mathrm{~km}$ mesh based on the $50 \mathrm{~m}$ mesh digital elevation data published by the Geographical Survey Institute.

The meteorological model targeted the Keihanshin region and the Kyushu region. Data for the windiest 15 days of each year were extracted from the wind data recorded over the past 20 years by the Japan Meteorological Agency (JMA) located within each region. Data for a total of 300 windy days were extracted. LOCALS calculated wind velocities for each grid point corresponding to a height of $10 \mathrm{~m}$ from the ground for every windy day.

\subsubsection{Initial and boundary conditions}

The reanalysis data prepared by the National Centers for Environmental Prediction (NCEP) and for Atmospheric Research (NCAR) were used to set the initial and boundary conditions for the numerical meteorological simulation. The intervals of approximately $250 \mathrm{~km}$ between grid points in the reanalysis data from NCEP/ NCAR could be considered fairly rough for the simulation, however, this reanalysis data did present the following advantages: (1)They were grid point values which could be easily processed in the numerical simulation, (2)They were already used by many other weather researchers in the world and were therefore dependable, (3)The reanalysis data covers the entire earth.

\subsubsection{LOCALS numerical simulation process}

LOCALS first calculated the evolution of metrological elements over time for a computational domain of $500 \mathrm{~km} \times 500 \mathrm{~km}$ on a $20 \mathrm{~km}$ mesh. The reanalysis data from NCEP/NCAR were used to set the initial and boundary conditions in this calculation. LOCALS then calculated the evolution of metrological elements over time for a computational domain of $20 \mathrm{~km} \times 20 \mathrm{~km}$ on a $3 \mathrm{~km}$ mesh. In this calculation, initial and boundary conditions were set according to the results of the first step in calculation. To satisfy the CFL condition (Courant-Friedrichs-
Lewy condition), time steps for meteorological simulation were set at 60 seconds (calculation on a $20 \mathrm{~km}$ mesh) and 10 seconds (calculation on a $3 \mathrm{~km}$ mesh). To obtain the daily maximum wind-velocity of each windy day, LOCALS carried out a calculation for a 27 hours period stretching from 21:00 of the previous day to 0:00 of the next day, and the maximum wind-velocity for each windy day was extracted from the calculation results obtained from LOCALS.

\subsection{Method for calculating the value of a return period}

This study adopts the modified Jensen \& Frank method [2,3] (hereafter, referred to as the MJF method) for calculating the value of the return period. Previous methods used by railways for this calculation (e.g., the Gumbel method) only used annual maximum wind-velocity data, whereas the MJF method can evaluate an extreme-value distribution not only by using the annual maximum wind-velocity data but also by using submaxima strong wind-velocity data. This means that the values over a given return period calculated with the MJF method and wind-velocity data observed even for a short period of time offers almost the same level of accuracy as the Gumbel method which relies on wind-velocity data observed over a long period of time.



1415161718192021222324252627282930 33 36 3940 Wind velocity $(\mathrm{m} / \mathrm{s})$

Fig. 2 Distribution for 50-year return period of mean windvelocity values at a point $10 \mathrm{~m}$ above the ground surface calculated by LOCALS and the MJF method 


\subsection{Results of mean wind-velocity value calculations for a given return period}

Wind velocity values were calculated for 50-year return period at a height of $10 \mathrm{~m}$ from the ground surface using the MJF method and daily maximum wind velocities calculated with LOCALS model. Figure 2 shows a distribution of those results in the Keihanshin area.

\section{Spatial distribution analysis of wind velocities using the computational fluid dynamics model}

\subsection{Computational fluid dynamics model}

\subsubsection{Overview of the computational fluid dynamics model}

In addition to the state of the atmosphere, topographical features influence wind-velocity. To investigate the influence of topography on airflow over several hundred meters in a scaled computational domain, we calculated mean wind-velocity distribution using a Computational Fluid Dynamics (CFD) model. CFD model reproduces wind direction, wind-velocity and atmospheric pressure at each grid point on a computational domain, utilizing fluid dynamics. The RIAM-COMPACT neutral stratification version CFD model developed by Research Institute for Applied Mechanics of Kyushu University [4] was used for this study. The RIAM-COMPACT model has the following features: (1)flexibility in calculation condition settings, (2)ability to adopt the Large Eddy Simulation (LES) model, which is appropriate for calculation of unsteady airflow on complex terrain, (3)efficient airflow calculation on computational domain ranging from a wide area to a local region using a nest-grid.

\subsubsection{Arrangement of grid points for airflow calculation with RIAM-COMPACT}

To obtain a detailed wind-velocity distribution, we set up a computational domain of 3 square kilometers with a grid point interval of 200 meters. Moreover, to reflect the influence of topographical features on the upstream side of the computational domain in the numerical simulation, the surrounding domain of 25 square kilometers was arranged outside the computational domain. The grid point intervals in the surrounding domain were set from 200 to 900 meters (nest-grid), increasing gradually in an outwardly direction. The height of computational domain was set at four times the height or more of the difference between the minimum elevation point and the maximum elevation point in the computational domain. The heights selected for the computational domain therefore included $2,000 \mathrm{~m}, 4,000 \mathrm{~m}$ or $6,000 \mathrm{~m}$ in each case. Effects of topographical features on wind-velocity vary depending on wind direction. Therefore, the RIAM-COMPACT carried out calculations for eight wind directions (North, North-East, East, South-East, South, South-West, West and NorthWest).

\subsubsection{Numerical simulation process under RIAM- COMPACT}

According to the initial condition of the set wind-velocity, the wind speed at the maximum elevation point in the computational domain was $15 \mathrm{~m} / \mathrm{s}$. The initial vertical wind-velocity profile followed a power law with a power index of 0.25 . The RIAM-COMPACT started calculation after setting of these wind-velocity distributions for the whole computational domain. The time step in the RIAMCOMPACT calculation was set at 0.05 second. Using the mean wind-velocity of main flow components from the calculation results of steps 50,000 to 60,000 level after which airflow steadied (hereafter referred to as the mesh mean wind-velocity), a wind-velocity ratio at a height of $10 \mathrm{~m}$ from the ground surface in the computational domain was calculated in the following way: mesh mean wind-velocity in the direction $\theta$ (= North, North-East, $\cdots$, North-West) at the grid point $(a, b)$ at $10 \mathrm{~m}$ above the ground surface is represented by $\bar{U}(a, b, \theta)$, the spatial average of $\bar{U}(a, b, \theta)$ in the computational domain of 3 square kilometers including the grid point $(a, b)$ is represented by $\bar{U}_{\text {area }}(\theta)$. The wind-velocity ratio $R_{200 m}(\theta)$ at the grid point $(a, b)$ is calculated by (1):

$$
R_{200 m}(a, b, \theta)=\bar{U}(a, b, \theta) / \bar{U}_{\text {area }}(\theta)
$$

\subsection{Calculation results of airflow distribution}

Figure 3 illustrates the wind-velocity ratio distribution at the height of $10 \mathrm{~m}$ above the ground surface obtained from calculation of the west wind using RIAMCOPACT.

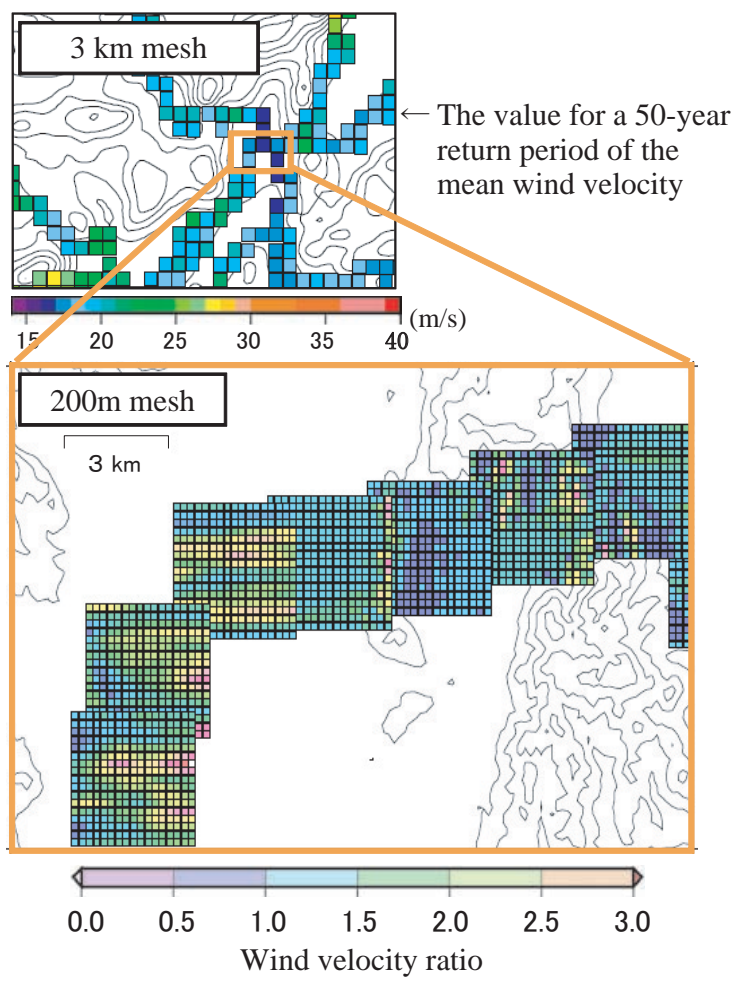

Fig. 3 Wind-velocity ratio distribution at a point $10 \mathrm{~m}$ above the ground surface calculated with RIAM-COPACT. 


\section{Estimation of gust factor using topographical factors}

This chapter describes a method for calculating the daily gust factor based on the topography and wind direction using multiple regression analysis. The daily gust factors by wind direction from meteorological observatories located in West Japan were used as the objective variable for the multiple regression analysis, and five kinds of topographical factor were used as explanatory variables.

\subsection{Daily gust factor}

The meteorological observatories in Japan release a daily maximum value for 10 -minute mean wind velocities and a daily maximum value of instantaneous windvelocity, both observed between 0 o'clock and 24 o'clock [5] (hereafter, the daily maximum wind-velocity and the daily maximum instantaneous wind-velocity, respectively). However, neither the daily maximum wind-velocity nor the daily maximum instantaneous wind-velocity is necessarily observed in the same ten minutes. We define the daily gust factor by the daily maximum instantaneous wind-velocity divided by the daily maximum wind-velocity, which is represented by $G_{d a y}$. The meaning of the value for the $N$-year return period of mean windvelocity can be interpreted as a magnitude of the daily maximum wind-velocity which can occur once in $N$ years. So, the value of mean wind velocities for an $N$-year return period multiplied by the daily gust factor $G_{d a y}$ can provide the value of the daily maximum instantaneous wind velocities for an $N$-year return period.

\subsection{Data used for topographical factor analysis}

The topographical features in the vicinity of 26 meteorological observatories located in West Japan were studied in the course of site investigations, surveys of topographical maps and documents. The wind directions where there were no obstructive factors such as buildings and trees in the way of wind observation were identified for each meteorological observatory. Data was extracted for windy days from April 1, 1989 to December 31,2005 , where the recorded daily maximum wind-velocity exceeded $10 \mathrm{~m} / \mathrm{s}$ in the identified wind directions in the absence of obstructive factors for wind observation. The daily maximum wind velocities and the daily maximum instantaneous wind velocities of those windy days for which data was extracted, were used to perform the topographical factor analysis.

\subsection{Daily gust factor per wind direction}

Daily gust factors were calculated from the same daily maximum wind velocities and the daily maximum instantaneous wind velocities employed in the previous section. The calculated daily gust factors classified into eight azimuth directions (e.g., the North corresponds to the range from 337.5 to 360 degree and 0 to 22.5 degree) based on the wind direction of the daily maximum wind velocities. JMA meteorological observatories however classify observational wind directions into 16 azimuth directions (e.g., the North corresponds to the range from 348.75 to 360 degree and 0 to 11.25 degree). As such, the north-northwest, north and north-north-east defined in the 16 azimuth directions were combined and redefined as the north in the eight azimuth directions [6]. The north-east, northwest etc in the eight azimuth directions were similarly defined by combining the corresponding three directions defined in 16 azimuth directions. Averaged daily gust factors per azimuth direction were adopted as the objective variable for the multiple regression analysis.

\subsection{Topographical factor by wind direction}

The topographical factor is an index indicating the features of on a terrain and it is calculated based on the elevation data at a grid point. The traditional method used in topographical factor analysis calculates the topographical factor within a radius of $R$ kilometers or for a squared area of $2 R$ kilometers of side length with the target point (nearing the meteorological observatory) at the center of the respective areas; this method does not consider azimuth directions. However, the topographical features located on the windward side of a meteorological observatory strongly influence the gust factor. Therefore, in addition to the traditional topographical factors in which azimuth directions are not taken into consideration, the topographical factors by azimuth directions were adopted as explanatory variables in the multiple regression analysis to estimate gust factors. Moreover, the roughness length and installation height of a wind vane and anemometer from the surface of the ground were added to the explanatory variables; the roughness length is an index that indicates ground surface conditions, and the installation height of a wind vane and anemometer above ground surface is a factor which strongly influences the gust factor. Nine topographical factors at each point were calculated: one was the topographical factor without consideration of azimuth direction, and the other eight ones were topographical factors for each of the eight azimuth directions. The installation heights of the wind vane and anemometer above the surface of the ground do not depend on azimuth direction. Figure 4 shows the area used to calculate the topographical factors by azimuth direction (hereafter, referred to as the factor calculation area). Length $R$ indicating triangular height was defined as an index representing the range of the effect of the terrain. Each topographical factor was calculated for four cases ( $R=1,3,5$, and 10 kilometers). The topographical factor without consideration of azimuth direction was calculated for the octagonal area comprising the eight isosceles triangles shown in Fig. 4.

$50 \mathrm{~m}$ mesh digital elevation data from the Geographical Survey Institute were used for calculating the topographical factors based on elevation (hill factor, openness factor, and land factor). A 100m mesh land-use database of the Ministry of Land, Infrastructure and Transport was used for calculating the roughness length. The topographical factors used for the analysis are described as follows:

\section{(1) The hill factor}

The hill factor was defined as standard deviation of the elevation of each grid point in the factor calculation 


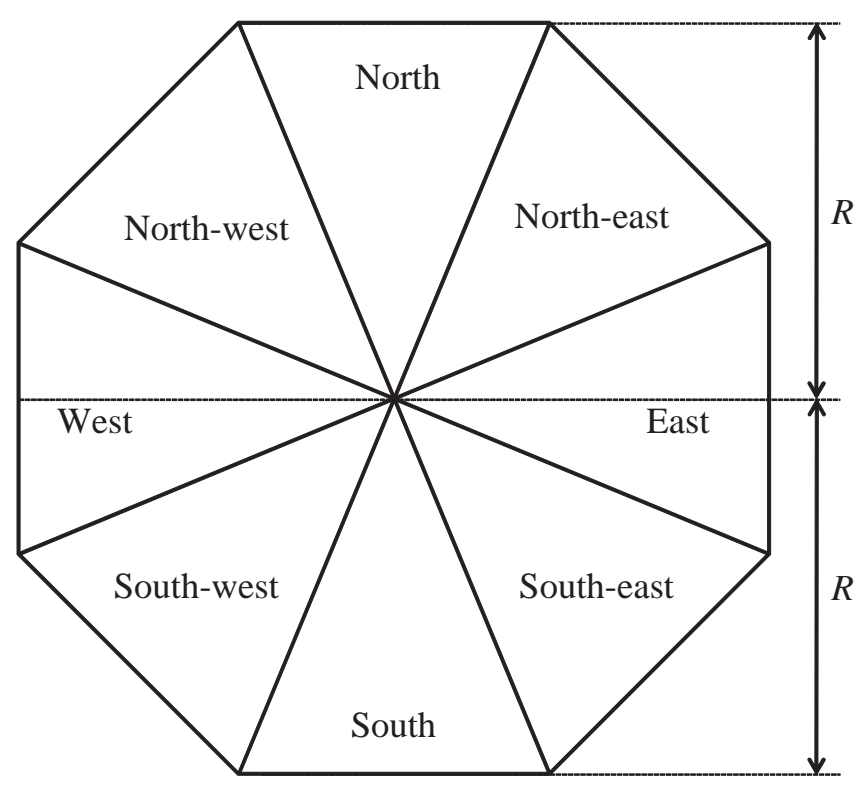

$R=1,3,5,10 \mathrm{~km}$

Fig. 4 Eight factor calculation areas

area, shown in Fig. 4. The elevation of the grid point $(a, b)$ in the azimuth direction $\theta$ and the average elevation in the factor calculation area are represented respectively by $h(a, b)$ and $\bar{h}$.

The hill factor $D(\theta, R)$ in the azimuth direction $\theta$ (north, north-east, $\cdots$, north-west and all azimuths) is represented by (2):

$$
D(\theta, R)=\frac{1}{M} \sqrt{\sum(h(a, b)-\bar{h})^{2}}
$$

Here, $M$ is a number of grid points in the factor calculation area.

\section{(2) The openness factor}

The definition of the openness factor is given as follows: a horizontal plane of $H+\Delta H$ of the elevation is assumed in the factor calculation area where $H$ represents the elevation of the target point, and $\Delta H$ has the value of $-200 \mathrm{~m},-100 \mathrm{~m}, 0 \mathrm{~m},+100 \mathrm{~m}$ or $+200 \mathrm{~m}$. The openness factor is defined as the ratio of the number of grid points positioned below the horizontal plane, (the elevation of $H+\Delta H$ ) to the number of all grid points in the factor calculation area. The openness factor $O P(\theta, \Delta H, R)$ in the wind direction $\theta$ is represented by ( 3$)$ :

$O P(\theta, \Delta H, R)=$

the number of grid points positioned below the horizontal

plane $(H+\Delta H)$ in the azimuth direction $\theta$

$$
M
$$

(3) The land factor

The land factor is defined as the ratio of the number of grid points of elevation 0 meters above sea-level or more (referred to as land), to the number of all grid points in the factor calculation area. The land factor $G_{r}(\theta, R)$ in the wind direction $\theta$ is represented by (4):
Table 1 Ground level conditions classified by the land-use database and corresponding roughness length

\begin{tabular}{l|l}
\hline $\begin{array}{c}\text { Condition of the ground level } \\
\text { according to the land-use database } \\
\text { classification }\end{array}$ & \multicolumn{1}{c}{ Roughness length (m) } \\
\hline \hline Rice field & 0.1 \\
\hline Agricultural land except rice field & 0.1 \\
\hline Forest & 1 \\
\hline Waste land & 0.25 \\
\hline Built area & 2 \\
\hline Transport use & 1 \\
\hline Other ground & 0.25 \\
\hline Rivers and lakes & 0.0005 \\
\hline Coastline & 0.01 \\
\hline Sea water & 0.0005 \\
\hline Golf field & 0.5 \\
\hline
\end{tabular}

$\operatorname{Gr}(\theta, R)=\frac{\text { the number of grid points of land }}{M}$

(4) The roughness length

The roughness length is an index that indicates the state of roughness on the ground surface, and is defined as the height where the wind-velocity becomes zero under the condition that the wind profile follows the logarithm rule represented by (5):

$$
U=\frac{u_{*}}{k} \ln \frac{z}{z_{0}}
$$

Where $U$ is the mean wind-velocity at the height $z, k$ is Karman constant, and $z_{0}$ is the roughness length. The roughness length was calculated based on the land-use database previously described. The land-use database classifies ground level conditions into 11 categories, shown in Table.1. A roughness length value was allocated to each land-use category, referring to Shiotani's book [7] and study cases in Europe [8]. The roughness length $z_{0}$ was obtained by averaging the values of the allocated roughness lengths in the factor calculation area.

(5) The installation height of wind vanes and anemometers above the surface of the ground

The installation height of wind vanes and anemometers above the surface of the ground for each meteorological observatory is represented by $z$.

\subsection{Daily gust factor estimation equation}

Based on the data obtained from the 26 meteorological observatories located in West Japan, a multiple regression analysis was performed with objective and explanatory variables defined as follows: the daily gust factor by wind direction was adopted as the objective vari- 
able, and four kinds of topographical factor and the installation height of wind vanes and anemometers above the surface of the ground were adopted as explanatory variables. First of all, the correlation coefficient between the daily gust factor and each explanatory variable and correlation coefficient between pairs of explanatory variables were calculated. The multiple regression analysis excluded explanatory variables with a correlation coefficient exceeding 0.6 , and was performed using remaining explanatory variables. This produced (6) for estimating the daily gust factor $G_{d a y}(\theta)$ in azimuth direction $\theta$.

$$
\begin{aligned}
& G_{\text {day }}(\theta)=0.44 \cdot \frac{1}{\ln \left(z / z_{0}\right)}-0.009 \cdot z \\
& -0.68 \cdot O P(\mathrm{ALL}, 200,10)+0.27 \cdot \operatorname{Gr}(\theta, 3)+2.34
\end{aligned}
$$

Where 'ALL' means 'all azimuth directions'. The correlation coefficient between the daily gust factor obtained from the observational wind data from the 26 meteorological observatories in West Japan and estimated using (6), was 0.67 .

\section{The wind-velocity return-period value along railway lines}

This chapter introduces the method for estimating the return period value for maximum instantaneous wind velocities along railway lines by using three values: the mean wind-velocity value for a return period calculated with the meteorological model and MJF method, the windvelocity ratio per wind direction calculated with the CFD model, and the daily gust factor obtained through multiple regression analysis. Here, the return period is assumed to be 50 years. First, the mean wind-velocity value for a 50-year return period at a height of $10 \mathrm{~m}$ from the ground surface was calculated using LOCALS and the MJF method expressed by $\bar{U}_{50 y}: \bar{U}_{50 y}$ means an expected value at a grid point that located nearest to the target point along the railway line. The distribution of the value for a return period of mean wind-velocity in $200 \mathrm{~m}$ mesh can be obtained by multiplying $\bar{U}_{50 y}$ by the wind-velocity ratio per wind direction $R_{200 m}(\theta)$ calculated using RIAMCOMPACT. The value for a return period with a mean wind-velocity $\bar{U}_{50 y, 200 m}(\theta)$ in the case where the same windvelocity as $\bar{U}_{50 y}$ is occurs in the wind direction $\theta$ can be written as follows.

$$
\bar{U}_{50 y, 200 m}(\theta)=\bar{U}_{50 y} \cdot R_{200 m}(\theta)
$$

The multiplication of $\bar{U}_{50 y, 200 \mathrm{~m}}(\theta)$ by the daily gust factor $G_{d a y}(\theta)$ at a height of $10 \mathrm{~m}$ above the ground surface $(z=$ 10 meters in (6)) obtained at intervals of $100 \mathrm{~m}$ produces the value for a return period of the maximum instantaneous wind velocities per wind direction at a height of $10 \mathrm{~m}$ from the ground surface at intervals of $100 \mathrm{~m}$. Here, the reason for $z=10 \mathrm{~m}$ in (6) was to enable the collation of values for a return period of maximum instantaneous wind velocities obtained by (7) with the wind data observed at weather stations, such as meteorological observatories, whose typical anemometer installation height is $10 \mathrm{~m}$. Next, a weighting per wind direction was added

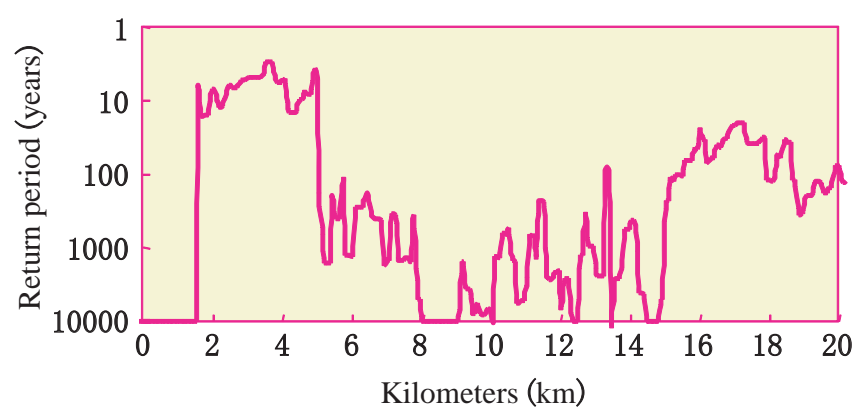

Fig. 5 An example of a return period with an annual maximum wind-velocity equal to $40 \mathrm{~m} / \mathrm{s}$ at a height of $10 \mathrm{~m}$ above the ground surface on virtual railway lines

to (7) using frequency of wind direction of strong winds calculated with LOCALS for the 300 windy days mentioned in Chapter 3: we counted the frequency of the maximum wind-velocity calculated in the wind direction $\theta$ at the grid point nearest to the target point, and divided this frequency by the total number of windy days, to obtain a weighting coefficient for wind direction $\theta$ at a given grid point. The weighting coefficient of the wind direction $\theta$ was represented by $D_{r}(\theta)$. Taking into consideration the daily gust factor $G_{d a y}(\theta)$ and weighting coefficient $D_{r}(\theta)$ into $(7)$, the value over a 50 -year return period of the maximum instantaneous wind-velocity at a height of $10 \mathrm{~m}$ from the ground surface at a certain grid point $\tilde{U}_{50 y}$ can be represented by (8):

$$
\tilde{U}_{50 \mathrm{y}}=\sum_{\theta} \bar{U}_{50 y, 200 m}(\theta) \cdot G_{d a y}(\theta) \cdot \operatorname{Dr}(\theta)
$$

Figure 5 shows an example for a return period, of the annual maximum wind-velocity equal to $40 \mathrm{~m} / \mathrm{s}$ at a height of $10 \mathrm{~m}$ from the ground surface for a virtual railway line.

\section{Conclusions}

This paper reports on a method to estimate the value for a return period of the maximum instantaneous windvelocity along railway lines at $100 \mathrm{~m}$ intervals using two kinds of numerical analysis (the meteorological model and the CFD model) and topographical factor analysis. The estimated value for a return period of the maximum instantaneous wind-velocity along railway lines obtained via this method can be used as an objective basis for optimum positioning of anemometers and reasonable settings of operational control sections. There are not many reports on the techniques for estimating the maximum instantaneous wind-velocity through numerical analysis. Moreover, verification of the values for a return period of wind velocities calculated in this study turned out to be a difficult problem. The authors hope to develop this technique in the future, by adoption of a new meteorological model and data assimilation technique, use of detailed reanalysis data, and subdivision of wind direction in the CFD model calculation and topographical factor analysis. 


\section{References}

[1] Tanikawa, R., "Development of the Wind Simulation Model by LOCALSTM and Examination of Some Studies," NAGARE, Vol.22, No.5, pp.405-415, 2003 (in Japanese).

[2] Cook,N.J., "Improving the Gumbel analysis by using M-th highest extremes," Wind and Structures, Vol.1, No.1, pp.25-48, 1998.

[3] Ohtake, K., Tamura, Y., "Study on estimation of flat terrain for each wind direction," Proceedings of Conference on Architectural Institute of Japan 2002, pp.119120, 2002 (in Japanese).

[4] Uchida, T., Ohta, Y., "Development of the Local Wind
Field Simulator RIAM-COMPACT -Wind Field Assessment and Real Time Simulation-," NAGARE, Vol.22, No.5, pp.417-428, 2003 (in Japanese).

[5] Japan Meteorological Agency, Guideline for surface weather observation, Japan Weather Association, 1993 (in Japanese).

[6] Architectural Institute of Japan, Recommendations for Loads on Buildings, Maruzen, 2004 (in Japanese).

[7] Shiotani, M., Characteristics of Strong Wind, KAIHATUSHA, 1979 (in Japanese).

[8] DB and SNCF, Common DEUFRAKO Research on Cross Wind Effects on High Speed Railway Operation 20012004, 2005. 